\section{May symptoms of chronic cerebrospinal venous insufficiency be improved by venous angioplasty? An independent 4-year follow up on 366 cases}

\section{Pietro M. Bavera}

Vascular Surgeon at the University of Milano; Vascular Imaging Diagnostician for Medick-Up Vascular Lab, Milano, Italy

\section{Abstract}

The aim of the study was to collect results from 366 chronic cerebrospinal venous insufficiency (CCSVI) affected patients that were regularly Duplex controlled after having received vein angioplasty following diagnosis for CCSVI. The procedures were all performed in the same Centre and same equipment. The patients were divided into three groups according to the attributed severity of the associated multiple sclerosis: 264 relapse-remitting (RR) (72\%): 179 females $(67.8 \%)$ and $85(32.2 \%)$ males; 62 secondary progressive (17\%): 37 (59.7\%) females and 25 (40.3\%) males; 40 primary progressive (11\%): 22 (55\%) females and 18 (45\%) males. A data base revealed eleven most frequent disturbs and symptoms, together with working capacities, and was kept up-todate at every Duplex control aiming to establish a novel rapid CCSVI symptoms questionnaire assessment in 4 years follow up. The symptoms were: diplopia, fatigue, headache, upper limb numbness/mobility, lower limb numbness/mobility, thermic sensibility, bladder control, balance coordination, quality of sleep, vertigo, mind concentration.

Results, as follows, appear to be significantly good in the RR group, also the biggest one. Diplopia improved in 262/264 patients (99.2\%) $(\mathrm{P}<0.0001)$; fatigue in $260 / 264 \quad(98.5 \%)$ $(\mathrm{P}<0.0001)$; headache in $205 / 208(98.6 \%)$ $(\mathrm{P}<0.0001)$; balance coordination in $23 / 26$ (88.5\%) $(\mathrm{P}<0.0001)$; quality of sleep in $55 / 59$ (93.2\%) $(\mathrm{P}<0.0001)$; vertigo in $30 / 33(90.9 \%)$ $(\mathrm{P}<0.0001)$; mind concentration in $142 / 144$ (98.6\%) $(\mathrm{P}<0.0001)$. Other results regarded: upper limb numbness and mobility in 20/24 (83.3\%) ( $\mathrm{P}=0.0002)$; lower limb numbness and mobility $13 / 15(86.7 \%)(\mathrm{P}=0.0087)$; thermic sensibility $3 / 4 \quad(75 \%)$ [P: not significant (n.s.) ]; bladder control 2/3 (66.6\%) (P: n.s.).

In contrast in the progressive cases results are quite different where, nevertheless, some useful considerations were collected and statistically significant, too.

In addition, venous angioplasty appears to be safe, side effects were observed only in seven patients $(0.19 \%)$ that grew a monolateral Jugular thrombosis but still were regularly controlled and above all did not suffer worsening of the disease.

Finally, the overwhelming percutaneous transluminal angioplasty results in the RR group lead to say that the correct criteria should be the sooner the better.

\section{Introduction}

Chronic cerebrovascular venous insufficiency (CCSVI) is perhaps one of the most important new chapters in vascular diseases, often correlated with severe neurologic ones, of the past decade. Certainly it is one of the most frequent discussion topics related to venous malformations. Moreover, this argument has openly split medical opinions and shaken a worldwide population of patients affected with multiple sclerosis (MS). ${ }^{1}$ All this turmoil started a few years ago, in 2007, when Prof. Paolo Zamboni first presented his paper regarding the $B$ ig Idea ${ }^{2}$ that gave fire to the fuse and shortly after exploded with shattering effects inside medical scientific world. ${ }^{3-6}$

In past, we gave our contribution with two papers, ${ }^{7,8}$ with the purpose to realize the presence of CCSVI in MS affected patients through Duplex exams. In that occasion the purpose of the exams was principally to find out possible presence of haemodynamic vascular irregularities (stenosis and/or valve malfunctions) ${ }^{9-11}$ regarding venous outflow from the brain stream and cervical medulla. Since the beginning of all, the definition for CCSVI and the protocol for carrying out this type of diagnostic exam was defined by Menegatti et al. ${ }^{12,13}$ and still is the main reference in this argument.

Since 2007 and onwards, hundreds of papers were written on this argument and divided schools of thoughts, and now even some textbooks are introducing the pathology.

After having achieved the correct know-how directly from Zamboni in mid 2010, the author started in November 2010 to examine MS patients, and is still doing so at present.

After four years, and completed exam 2150, the collected results were sorted-out and the present study is addressed in assessing the effect of percutaneous transluminal angioplasty on the more frequent and disabling symptoms coming from the association between CCSVI and MS.

\section{Materials and Methods}

The work regards 366 MS affected patients, 238 females and 128 males between 18 and 58
Correspondence: Pietro M. Bavera, Vascular Surgeon at the University of Milano; Vascular Imaging Diagnostician for Medick-Up Vascular Lab, Milano, Italy.

E-mail: pietromaria.bavera@fastwebnet.it

Conflict of interest: the author declares no potential conflict of interest.

Acknowledgments: the author would like to thank Dr. Nicolò Margaritella for his valuable help in statistical evaluations.

Key words: Chronic cerebrospinal venous insufficiency; multiple sclerosis; jugular veins; venous angioplasty; symptoms.

Received for publication: 26 June 2015 .

Revision received: 15 September 2015.

Accepted for publication: 17 September 2015.

This work is licensed under a Creative Commons Attribution 3.0 License (by-nc 3.0).

(C) Copyright P.M. Bavera, 2015

Licensee PAGEPress, Italy

Veins and Lymphatics 2015; 4:5400

doi:10.4081/vl.2015.5400

years old (mean age 33.3), that were both interviewed and Duplex examined between November 2010 and December 2014, growing a totality of 1464 consecutive Duplex exams. All duplex control exams were, every time, anticipated by a regular interview with standardized questions with the aim to seek and register eventual changes of symptoms in a certain lapse of time. The first word was therefore given to the patients and their answers allowed to collect objective clinical situations that were later compared with the Duplex exam result. The Author collected the eleven most frequent symptoms in these groups of patients and described them in another publication. $^{14}$

All scheduled controls were performed after venous angioplasty of one or both internal jugular veins and, when necessary, also of the azygos vein. In some occasions the venous PTA procedure was repeated a second or even third time but follow-ups were still based on the same number of controls within a four-year period.

All the Patients had a first Duplex exam, called exam zero, that was positive to CCSVI according to Zamboni's and the Consensus Document criteria ${ }^{15}$ and they autonomously underwent to vein PTA, freely choosing their Physician, either Vascular Surgeon or Interventional Radiologist.

The patients were divided into three groups according to the expanded disability status scale classification ${ }^{16}$ attributed by their Neurologists. Moreover, on the basis of the latest discharge letters, the patients' disease was 
sorted into: relapse-remitting (RR), secondary progressive (SP), and primary progressive (PP). These criteria also helped to obtain more homogenous results and comparisons.

In the RR group the patients were 264 (72\%), in the SP $62(17 \%)$ and in the PP 40 (11\%).

A demographic table describes each MS group with the clinical course age, expressed as mean and standard deviation and gender, male/female (Table 1).

The RR Patient group was the relatively easiest one to follow-up since 201 (76\%) underwent to only one angioplasty and $63(24 \%)$ to a second one, while none had a third procedure.

The SP Patient group resulted the most articulated and complicated since only 3 (5\%) stopped after one angioplasty while 51 (82\%) underwent to a second one and 8 (13\%) to a third procedure.

In the PP Patient group none (100\%) stopped after the first angioplasty and 37 $(92 \%)$ had a second one while only $3(8 \%)$ had a third procedure.

A peculiarity of the RR group was that the second angioplasty was always performed between 12 and 18 months after the first one while both SP and PP groups had a re-do in average 6 months after the first one and eventually a third procedure in average 9 months after the second one.

As a rule for all, the first Duplex control was usually carried out within three months after the first procedure.

The description of the venous PTA was read always after the Duplex exam, so to avoid any possible influence on the exam itself, making each time as if it was the first one. Moreover, each successive Duplex exam was performed without reading the results of previous ones.

All patients were first asked if they were willing to answer to standardized questions that regarded the most frequently past and eventually still present disorders in order to check-out for how long and how many benefits or improvements were active or wore-out in time. In order to help the patients and ease them, no particular scores were used from their answers but these were simply limited to catalogue the presence (+) or absence (-) of disorders in between a certain lapse of time after having received the angioplasty and also between the previous Duplex control.

The timetable for the controls was established after the first angioplasty and then quite flexible: within 3 months, 9/12 months, 18/24 months and lastly $36 / 48$ months. The Tables 2,3 and 4 regarding group divisions and Duplex exams compared with patient's answers are given with the results and only include those that underwent to regular Duplex controls.

This group of MS population established the definitive questionnaire list on the basis of 11 symptoms that were most frequently described. Other disorders or disabilities were less frequent and therefore discarded since probably would not have given significant numbers. These symptoms naturally were more persistent or consistent in parallel with the severity of the disease (SP and PP), while often happened to be absent in the less severe (RR) phase. Point 0 (zero), as also seen in the Tables, was considered as previous to the venous PTA. The eleven most frequently complained disorders or disabilities were: diplopia, fatigue, headache, upper limb numbness/ mobility, lower limb numbness/mobility, altered thermic sensibility, bladder control, balance coordination, quality of sleep, vertigo, mind concentration.

The working activity, secondary to the most common disabilities was included and influenced their symptomatology.

After having collected the information, the Duplex exam was performed. Usually, good Duplex results matched with clinical symptoms, especially in the RR group but did not always occur with the SP and PP figuring out that other situations could cause the disturbs. One among all often was a variable connected with ambulation difficulties and in general the use of devices to help walking. This particular situation apparently caused

Table 1. Patients' demographic characteristics stratified for multiple sclerosis course.

\begin{tabular}{lccc} 
MS course & No. & Age - mean (SD) & Gender F/M \\
RR & 264 & $28.5(5.3)$ & $179 / 85$ \\
SP & 62 & $47.9(7.1)$ & $37 / 25$ \\
\hline PP & 40 & $43.0(4.0)$ & $22 / 18$ \\
Total & 366 & $33.3(9.7)$ & $238 / 128$ \\
\hline
\end{tabular}

MS, multiple sclerosis; SD, standard deviation; F, female; M, male; RR, relapse-remitting; SP, secondary progressive; PP, primary progressive.

Table 2. Results on the relapse-remitting group of patients.

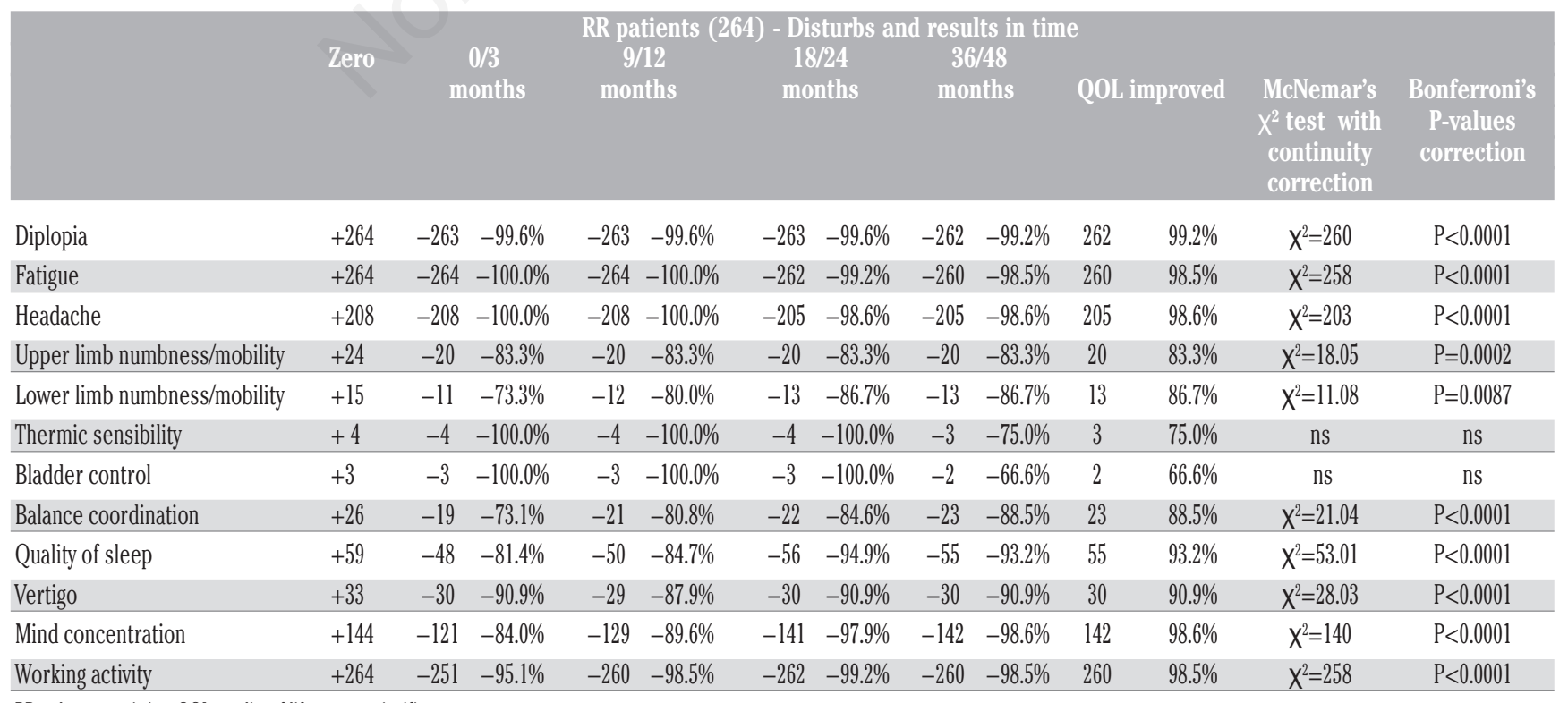

RR, relapse-remitting; QOL, quality of life; ns, not significant. 
an abnormal muscular fatigue of the neck, often with a sort of muscle hypertrophy, and in some cases to unnatural extrinsic compression of blood vessels.

Lastly, regarding Tables 2-4, statistical analyses were conducted using McNemar's test to determine whether the number of patients within each disorder or disability significantly changed between baseline (zero) and the last follow-up (36/48 months). Continuity correction to McNemar's test was applied to cope with disturb categories within which a low number of subjects were showed. P-values were multiple comparisons corrected using Bonferroni's procedure.

\section{Results}

The results, updated to the end of December 2014, regard a four-year follow-up. All controlled subjects underwent to venous angioplasty, no stentings were observed. The Duplex exams were always well accepted, appeared obviously safe and had an important role in the evaluation of the venous haemodynamic situation, ${ }^{17}$ combined with the purely CCSVI symptoms questionnaire as described by the patients themselves.

Firstly, after having clearly identified the presence of CCSVI in a considerable majority of patients affected by MS, there seems to be a frequent parallelism between some disturbs and the vein abnormalities: they usually improve or even disappear when the outflow is corrected. Secondly, both the clinical and blood flow improvements appear to be long lasting when they are not yet so severe and apparently the neurological damage is not so widely distributed.

This could explain why better and perjuring results appear to occur in the RR group of patients.

For the same reason this could be why the results are less stable in the more severe SP and PP patients that, nevertheless, still want to

Table 3. Results on secondary progressive patients.

\begin{tabular}{|c|c|c|c|c|c|c|c|c|c|c|c|c|c|}
\hline & & & & patier & nts (62) - & istur & $s$ and re: & ults il & time & & & & \\
\hline & Zero & & $\begin{array}{c}0 / 3 \\
\text { nonths }\end{array}$ & & $\begin{array}{l}\text { /12 } \\
\text { onths }\end{array}$ & & $\begin{array}{l}3 / 24 \\
\text { inths }\end{array}$ & & $\begin{array}{l}148 \\
\text { iths }\end{array}$ & QOL i & nproved & McNemar's & Bonferroni's \\
\hline Diplopia & +62 & -62 & $-100.0 \%$ & -62 & $-100.0 \%$ & -53 & $-85.5 \%$ & -49 & $-79.0 \%$ & 49 & $79.0 \%$ & $X^{2}=47.02$ & $\mathrm{P}<0.0001$ \\
\hline Fatigue & +62 & -60 & $-96.8 \%$ & -42 & $-67.7 \%$ & -15 & $-24.2 \%$ & -4 & $-6.5 \%$ & 4 & $6.5 \%$ & $X^{2}=2.25$ & $P>0.9$ \\
\hline Headache & +53 & -53 & $-100.0 \%$ & -52 & $-98.1 \%$ & -51 & $-96.2 \%$ & -50 & $-94.3 \%$ & 50 & $94.3 \%$ & $X^{2}=48.02$ & $\mathrm{P}<0.0001$ \\
\hline Upper limb numbness/mobility & $y+62$ & -61 & $-98.4 \%$ & -58 & $-93.5 \%$ & -46 & $-74.2 \%$ & -35 & $-56.5 \%$ & 35 & $56.5 \%$ & $X^{2}=33.03$ & $\mathrm{P}<0.0001$ \\
\hline Lower limb numbness/mobility & $y+62$ & -62 & $-100.0 \%$ & -31 & $-50.0 \%$ & -18 & $-29.0 \%$ & -10 & $-16.1 \%$ & 10 & $16.1 \%$ & $X^{2}=8.1$ & $\mathrm{P}=0.0531$ \\
\hline Thermic sensibility & +62 & -62 & $-100.0 \%$ & -55 & $-88.7 \%$ & -41 & $-66.1 \%$ & -12 & $-19.4 \%$ & 12 & $19.4 \%$ & $X^{2}=10.08$ & $\mathrm{P}=0.018$ \\
\hline Bladder control & +56 & -55 & $-98.2 \%$ & -54 & $-96.4 \%$ & -53 & $-94.6 \%$ & -46 & $-82.1 \%$ & 46 & $82.1 \%$ & $X^{2}=44.02$ & $\mathrm{P}<0.0001$ \\
\hline Balance coordination & +62 & -61 & $-98.4 \%$ & -31 & $-50.0 \%$ & -25 & $-40.3 \%$ & -15 & $-24.2 \%$ & 15 & $24.2 \%$ & $X^{2}=13.07$ & $\mathrm{P}=0.004$ \\
\hline Quality of sleep & +56 & -56 & $-100.0 \%$ & -50 & $-89.3 \%$ & -50 & $-89.3 \%$ & -50 & $-89.3 \%$ & 50 & $89.3 \%$ & $X^{2}=44.02$ & $\mathrm{P}<0.0001$ \\
\hline Vertigo & +52 & -51 & $-98.1 \%$ & -48 & $-92.3 \%$ & -26 & $-50.0 \%$ & -25 & $-48.1 \%$ & 25 & $48.1 \%$ & $X^{2}=23.04$ & $\mathrm{P}<0.0001$ \\
\hline Mind concentration & +62 & -62 & $-100.0 \%$ & -58 & $-93.5 \%$ & -57 & $-91.9 \%$ & -50 & $-80.6 \%$ & 50 & $80.6 \%$ & $X^{2}=48.02$ & $\mathrm{P}<0.0001$ \\
\hline Working activity & +62 & -62 & $-100.0 \%$ & -27 & $-43.5 \%$ & -14 & $-22.6 \%$ & -5 & $-8.1 \%$ & 5 & $8.1 \%$ & $X^{2}=3.2$ & $\mathrm{P}=0.884$ \\
\hline
\end{tabular}

SP, secondary progressive; QOL, quality of life.

Table 4. Results on primary progressive patients.

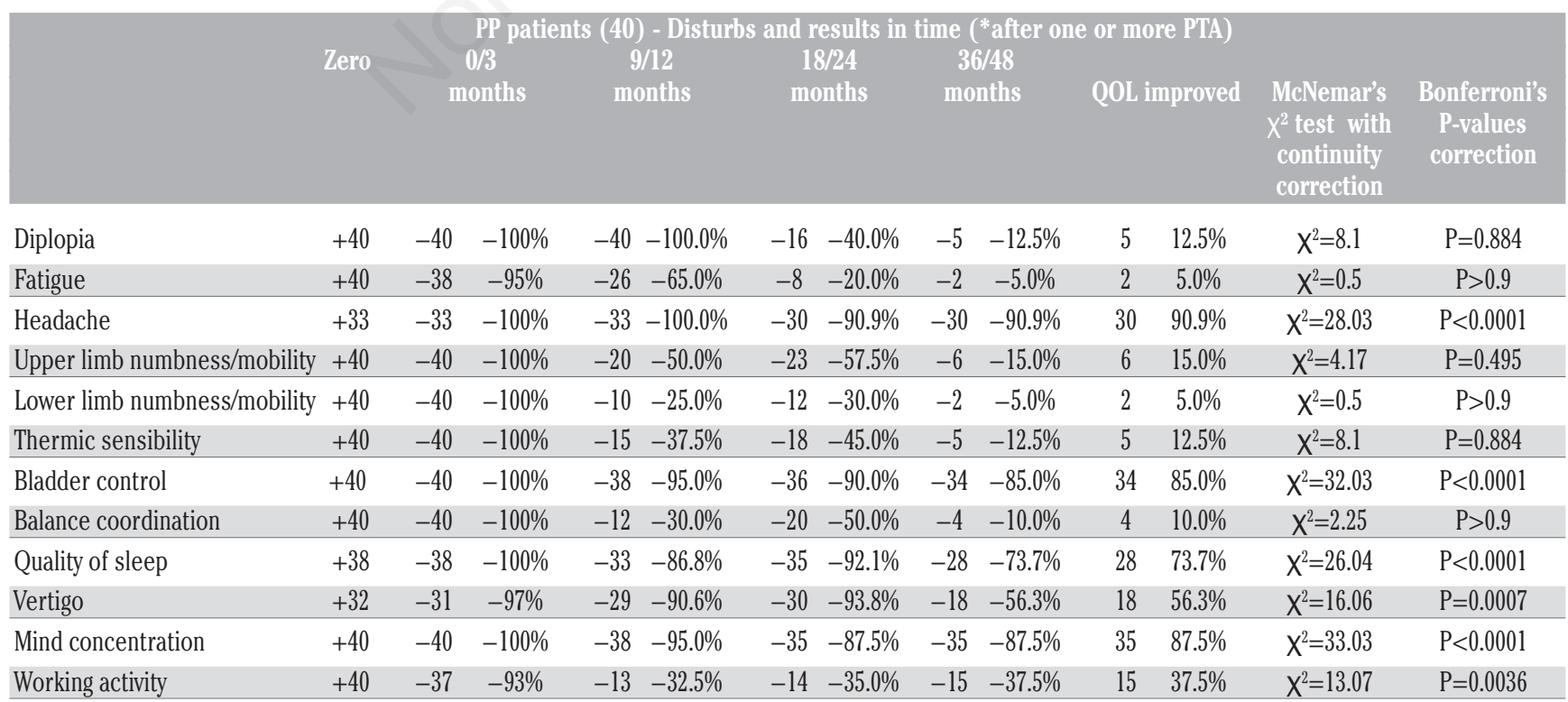

PP, primary progressive; PTA, percutaneous transluminal angioplasty; QOL, quality of life. 
try one or two re-do procedures.

Besides this, in these two latter groups of patients, mostly PP, the presence of external vein compression appears to influence the PTA and, in this group of examined patients, seems principally to occur on males.

The most important aspect, that regards the clinical disturbs, is that there is a matching Duplex exam: an improved bloodstream outflow corresponds with a better clinical situation while, on the other hand, one or more severe venous outflow problems meet with symptomatic disturbs that gradually return to a situation prior venoplasty.

Tables 2-4 are descriptive for disturbs and results.

Under a statistical point of view, the venous outflow problems re-occur most frequently in the SP and PP patients, usually found at the brachial cephalic junction and, most commonly, on the left side (72\%) otherwise bilaterally (21\%) while apparently less frequent is the monolateral right side alone (7\%). Valve leaflet restenosis alone appear to be more frequent in RR patients (91\%), while conjunct valve defects and vein stenosis mostly occur with SP (63\%) and PP (87\%) patients.

Working activities after three months in RR patients improved in $95.1 \%$ of cases and showed to be even better at the end of the survey $(98.5 \%)$.

In seven progressive patients, in this case always after control of the second procedure, a thrombosis of the internal jugular vein was observed, in six cases on the left side. These veins were all hypoplasic since before any whatsoever procedure and probably for this reason important haemodynamic changes never occurred. It was not therefore so strange, that the opposite side appeared to be giving a satisfactory compensation. Therefore, the left jugular district appeared to be most vulnerable, in this series of controlled patients.

In this series, symptomatology usually was the reason that leads these patients to submit themselves to venous angioplasty, more than a real attitude against traditional medical therapy. Disturb symptoms appear considerably improved in RR patients, as clearly evident in Table 2. Symptomatology appeared to be more complicated in the two progressive groups, as shown in Tables 3 and 4, where the amount of disturbs were already more present since starting point. Motion problems associated with extreme tiredness and quick exhaustion were the principal problems that lead to the venous angioplasty.

Peculiarity of the majority of this group was, in both cases of progressive forms, an extraordinary benefit that came in reduced symptoms (99.08\% for the SP and $99.27 \%$ for the PP) immediately after the venoplasty.

Most of the improvements in these two groups of patients unfortunately lasted for a short lapse of time, between two weeks and three months.

Veins and valve leaflets most probably recovered a sort of memory effect and this gradually overtook the venous angioplasty.

Moreover a general poor mobility and tremendous muscular stress required to keep sufficient balance during ambulation, usually with the help of devices, gradually developed and could be in some cases the cause of muscular hypertrophy of the neck and consequent extrinsic vein compression.

\section{Discussion}

This is an independent four-year study carried out on 366 MS patients with a regular follow-up plan. There is not the will to convince that CCSVI is the therapy for MS.

In this series it is quite evident that the positive results are observed in almost totality of relapse-remitting population and appear more persistent.

Again, the majority of progressive MS patients experienced, perhaps only for a short period, symptom improvements and for this reason submitted themselves to a second or even third procedure hoping to re-establish them. Anyhow, it is a matter of fact that both Duplex and clinical improvements were established in a sufficient number of treated patients with balloon angioplasty, enough to make this type of treatment as sufficiently safe, considering the low number of sideeffects observed or described in my series and confirmed in literature. ${ }^{18,19}$

Finally, as described in the most recent Consensus Document, ${ }^{20}$ parallelism between poor cephalic venous outflow and a list of symptoms, either neurologic or motor are confirmed. Symptoms appear to improve with a better venous outflow and the patients were very precise on this topic.

This work is autonomous, free from economical interests, based on the responsible help of all those Patients that helpfully answered in the past four years to questions collected at Duplex exams or controls, before and after venoplasty. For obvious reasons they are all kept anonymous but they were all informed that their voluntary help would become useful for this purpose.

\section{References}

1. Zamboni P, Menegatti E, Occhionorelli S, et al. The controversy on chronic cerebrospinal venous insufficiency. Veins and Lymphatics 2013;2:e14.

2. Zamboni P. The Big Idea Iron-dependent inflammation in venous disease and proposed parallels in multiple sclerosis. $\mathrm{J} \mathrm{R}$ Soc Med 2006;99:5.

3. Zamboni P, Galeotti R. The chronic cerebrospinal venous insufficiency syndrome. Phlebology 2010;25:269-79.

4. Zamboni P, Menegatti E, WeinstockGuttman B, et al. The severity of chronic cerebrospinal venous insufficiency in patients with multiple sclerosis is related to altered cerebrospinal fluid dynamics. Funct Neurol 2009;24:133-8.

5. Zamboni P, Morocic S, Menegatti E, et al. Screening for chronic cerebrospinal venous insufficiency (CCSVI) using ultrasound - Recommendations for a protocol. Int Angiol 2011;30:571-97.

6. Simka M, Kostecki J, Zaniewski M, et al. Extracranial Doppler sonographic criteria of chronic cerebrospinal venous insufficiency in the patients with multiple sclerosis. Int Angiol 2010;29:109-14.

7. Bavera PM, Mendozzi L, Cavarretta R, et al. Venous extracranial Duplex ultrasound and possible correlations between multiple sclerosis and CCSVI: an observational study after 560 exams. Acta Phlebologica 2011;12:109-13.

8. Bavera PM, Agus GB, Alpini D, et al. Results from 823 consecutive duplex exams for CCSVI in a vascular centre. Acta Phlebologica 2012;13:14-48.

9. Simka M, Kostecki J, Zaniewski M, et al. Extracranial Doppler sonographic criteria of chronic cerebrospinal venous insufficiency in the patients with multiple sclerosis. Int Angiol 2010;29:109-14.

10. Menegatti E, Tessari M, Gianesini S, et al. Human internal jugular valve M-mode ultrasound characterization. Curr Neurovasc Res 2013;11:149.

11. Zamboni P, Tisato V, Menegatti E, et al. Ultrastructure of internal jugular vein defective valves. Phlebology 2014 [Epub ahead of print].

12. Menegatti E, Genova V, Tessari M, et al. The reproducibility of colour Doppler in chronic cerebrospinal venous insufficiency associated with multiple sclerosis. Int Angiol 2010;29:121-6.

13. Menegatti E, Galeotti R, Gianesini S, et al. Echo-color-Doppler criteria for diagnosis of chronic cerebrospinal venous insufficiency. XVI World Congr UIP 2009. Int Angiol 2009;28:36.

14. Bavera P. The most frequent symptoms and disturbs from CCSVI. Behav Neurol 2015 [In press].

15. Lee BB, Bergan J, Gloviczki P, et al. Diagnosis and treatment of venous malformations. Consensus document of the International Union of Phlebology (UIP) 2009. International Union of Phlebology (UIP) Int Angiol 2009;28:434-51. 
16. Kurtzke JF. Rating neurologic impairment in multiple sclerosis: an expanded disability status scale (EDSS). Neurology 1983;33:1444-52.

17. Dolic K, Siddiqui AH, Karmon Y, et al. The role of noninvasive and invasive diagnostic imaging techniques for detection of extra-cranial venous system anomalies and developmental variants. BMC Med

\section{3;11:155.}

18. Hubbard D, Ponec D, Gooding J, et al. Clinical improvement after extracranial venoplasty in multiple sclerosis. Vasc Interv Radiol 2012;23:1302-8.

19. Zamboni P, Galeotti R, Weinstock-Guttman $\mathrm{B}$, et al. Venous angioplasty in patients with multiple sclerosis: results of a pilot study. Eur J Vasc Endovasc Surg
2012;43:116-22.

20. Lee BB, Baumgartner I, Berlien P, et al. Diagnosis and Treatment of Venous Malformations Consensus Document of the International Union of Phlebology (IUP): updated 2013. Int Angiol 2015;34:97-149. 